Rapid Communications

\title{
Pattern Recognition Analysis for Classification of Hypertensive Model Rats and Diurnal Variation Using ${ }^{1} \mathrm{H}-\mathrm{NMR}$ Spectroscopy of Urine
}

\author{
Masako FuJIWARA, ${ }^{* \dagger}$ Kazunori ARIfUKU, ${ }^{*}$ Itiro Ando,** and Tadashi Nemoto $* * * *$ \\ *JEOL DATUM LTD, 1156, Nakagami-cho, Akishima-shi, Tokyo 196-0022, Japan \\ **Environmental Research Center Ltd., 3-1, Hanari, Tsukuba-shi, Ibaraki 305-0857, Japan \\ ***National Institute of Advanced Industrial Science and Technology (AIST), \\ 1-1, Higashi, Tsukuba-shi, Ibaraki 305-8566, Japan
}

\begin{abstract}
Urine samples were collected during the daytime and nighttime from spontaneously hypertensive model rats and normal rats without dosing. The ${ }^{1} \mathrm{H}$ NMR spectra were measured for their urine samples, and analyzed by a pattern recognition method, known as Principal Component Analysis (PCA) and Soft Independent Modeling of Class Analogy (SIMCA). The separation of urinary data due to the diurnal variation (daytime and nighttime) and also to the difference between the two strains of rat was achieved in the PCA score plot. Differences of the urinary profiles in the respective separation were effectively extracted as marker variables by the SIMCA method. NMR measurements coupled with pattern recognition methods provide a straightforward approach to inspect the disease metabolic status and the preliminary screening tool of marker candidates for further development.
\end{abstract}

(Received August 31, 2005; Accepted September 28, 2005)

\section{Introduction}

NMR-based Metabolomic analysis has mainly been used for toxicology or drug discovery. ${ }^{1,2}$ Significant metabolic responses have been detected on various bio samples with the dose, injection of toxins or high-salt diet. Recently, various life-style related diseases, such as hypertension, diabetes, and hyperlipidemia, have become serious topics of concern. To acquire potential information on disease status, including presymptomatic data, the detection of any profile difference from the normal in natural conditions is essential. To understand the progress of disease metabolism, we performed an experiment with 20 model rats: 10 rats that were spontaneously hypertensive (SHR/Izm) and 10 rats that were normal (Wistar Kyoto, WKY). The two strains' urine samples were collected under conditions of minimized intentional stress, such as on impact, dose, special diet and fasting. ${ }^{3,4}$ The 1D ${ }^{1} \mathrm{H}-\mathrm{NMR}$ spectra for the urine samples were measured. All of the spectra were analyzed by a pattern recognition method and classified by their pattern differences. It is notable that there is no need to assign each peak or to identify the compounds in the spectrum, which can be recognized as a profile histogram..$^{5}$ To investigate the reflection of the metabolic balance in excretions, the pattern recognition method is a faster and more straightforward approach than conventional NMR structure analysis.

\section{Experimental}

Animal handling and sample collection

Ten rats each of 9-week-old males of WKY and SHR/Izm

† To whom correspondence should be addressed.

E-mail: masako@jeol.co.jp strain were purchased from SHIMIZU Laboratory Supplies Co., Ltd. The rats were housed by Bio-Science Center of KAC Co., Ltd. (Kyoto, Japan) in metabolic cages for 1 week acclimatization. All of the studies were conducted using the same basic protocol in that a standard solid diet, CRF-1 (Oriental Yeast Co. Ltd.) was given to all animals and free access to food and water was permitted. A temperature of $23 \pm$ $2^{\circ} \mathrm{C}$ and a relative humidity of $50 \pm 10 \%$ were maintained with a $12 \mathrm{~h}$ light/12 h dark cycle. Urine samples were collected from each 10-week-old of WKY strain (average b.p. 105/75 mmHg, wt $265 \mathrm{~g}$ ) and SHR/Izm strain (average b.p. 171/140 mmHg, wt $255 \mathrm{~g})$, during the daytime (14:00 - 18:00) and nighttime (18:00 - 9:00), respectively. The samples were sent to us maintained at $0-4^{\circ} \mathrm{C}$, and then measured within 2 days. In our laboratory, after centrifuging, $200 \mu \mathrm{L}$ of a phosphate buffer solution was added to $400 \mu \mathrm{L}$ of rat urine, with which $5 \% \mathrm{D}_{2} \mathrm{O}$ and $0.1 \%(\mathrm{w} / \mathrm{v}) \mathrm{NaN}_{3}$ were mixed, to obtain $\mathrm{pH}$ 7.4. Each 600 $\mu \mathrm{L}$ of the solution was used for an NMR measurement.

\section{NMR spectroscopy}

The ${ }^{1} \mathrm{H}$ NMR spectra were acquired using a $500 \mathrm{MHz}$ JEOL ECA spectrometer at $25^{\circ} \mathrm{C}$. Each spectrum consisted of $16 \mathrm{~K}$ complex data points with a spectrum width of $5 \mathrm{kHz}$, where each spectrum was accumulated by 128 scans with an acquisition time of $1.75 \mathrm{~s}$, and a recycle delay of $5 \mathrm{~s}$ per scan. The detection pulse flip angle was set to $45^{\circ}$. A pre-saturation sequence was used to suppress the water signal.

NMR data reduction procedures and pattern recognition analysis Each NMR spectrum was segmented into 225 regions of 0.04 ppm width over a range of 0.5 to $9.5 \mathrm{ppm}$, and each segment of the spectral regions was integrated. Integrated regions from 4.5 to $6.0 \mathrm{ppm}$, which contained both water and urea signals, were eliminated from the data table, and then the data were reduced to 188 variable regions. ${ }^{5}$ The remaining integral values of each 


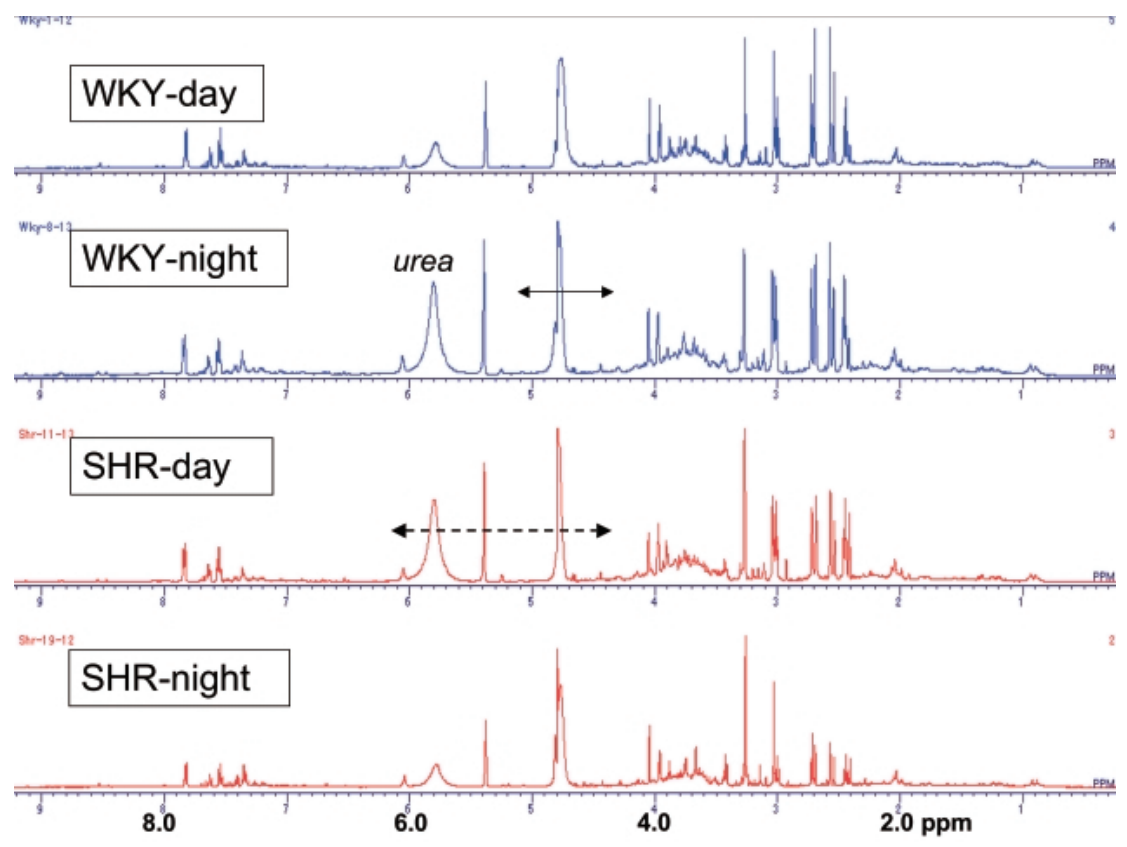

Fig. 1 Examples of raw ${ }^{1} \mathrm{H}$ spectra of urine samples. From the top: WKY day, WKY night, SHR day and SHR night, respectively. The regions of resonance, indicated by dashed arrow, including residual water (the bold arrow) and urea (broad signals), were eliminated from the dataset.

spectrum were normalized for 100 of the total summed integrals in order to compensate for any differences of the concentration between urine samples. Spectral processing was performed by 'ALICE 2 for Metabolome' (JEOL) version 1.0 software $^{6}$ and successive pattern recognition analysis was performed using Sirius (PRS, Norway) version 6.5 software.

The primary tool applied for pattern recognition analysis was PCA, with visualization of the PCA scores representing the main interaction. ${ }^{5}$ Based on the results from PCA, a 'supervised' pattern recognition model was developed using the SIMCA algorithm. ${ }^{7-9}$ The Cooman's plot is a good way to visualize the SIMCA approach for the two models. Its advantage is that SIMCA can generate the discrimination power by variables that contribute to classifying the two models.

\section{Results}

${ }^{1} H$ spectra of each strain at daytime and night

Figure 1 shows the ${ }^{1} \mathrm{H}$ NMR spectra of each example of WKY and SHR during the daytime and nighttime, respectively. The assignment of signals in normal rat urine has been mentioned elsewhere. ${ }^{10}$ In this study the whole pattern balance was to be investigated. While the spectral patterns of the four examples appear to be similar, as in Fig. 1, the classification used to discern the sample categories is possible by following analyses.

\section{Pattern recognition analysis: I) PCA-method}

Three daytime samples were not collected, and one WKY rat was recognized as an outlier and discarded by a preliminary statistical analysis. Therefore, a total of 35 spectra were submitted to PCA, in which all of the 188 variables were equally accounted for the data sets (not standardized). The result of the PCA score plot of PC1 and PC2 is shown in Fig. 2. The score sum of PC1 and PC2 was 78.6\%, which explains the characteristics of the samples sufficiently. The four spectra in Fig. 1 are indicated in Fig. 2.
Four clear classifications appear in Fig. 2: the samples of WKY during the daytime (blue circles) and nighttime (blue squares), SHR during the daytime (red circles) and nighttime (red squares). This can also be interpreted as two ways for two separate classifications:

(1) All of WKY (blue) and that of SHR (red) seem to be separate, which is the difference between the two strains.

(2) All of daytime (circles) and that of nighttime (squares) seem to be separate, which is the diurnal variation.

To confirm the reproducibility, four randomly selected samples were measured again after $10 \mathrm{~h}$, and a total of 39 samples were submitted to PCA again; in the score plot each pair was identified (Fig. 3), which resulted in good reproducibility.

\section{Pattern recognition analysis: II) SIMCA-method}

In this section, we describe how two types of classification mentioned in the previous section are inspected by a secondary tool for pattern recognition by the SIMCA method. First, regarding classification (1), for all WKYs and all SHRs, independent mathematical models (note that the 'model' used here is a mathematical one, and not the disease animal model) were made by applying PCA, and submitted to the SIMCA. Figure 4 shows the results of SIMCA, a Cooman's plot and a discrimination power plot of both models via 188 variables. Each sample is plotted in the Cooman's plot, where the distances are horizontal and vertical vectors from each model. Samples of SHR and WKY are almost within their $68 \%$ confidence limit, respectively, in Fig. 4. This means that a good classification resulted. The variables with higher discrimination powers than 1.0 contribute to discern the two. The discrimination powers here were not much higher than 1.0, mostly, which means that the entire spectral profiles of SHRs and WKYs resemble each other, as shown in Fig. 1. However, it is remarkable that some variables with higher discrimination power are distinct markers correlating with the difference between the two strains. The variables with discrimination powers higher than 2.0 were $2.41,2.58,2.70$, and $3.27 \mathrm{ppm}$, 


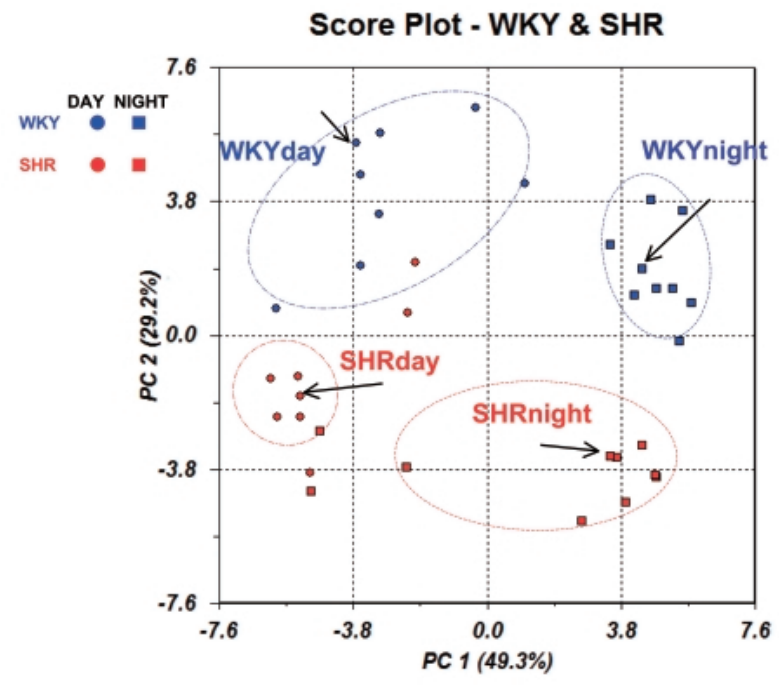

Fig. 2 PCA score plot of the first two PCs for all samples. The blue and red colors indicate WKY and SHR samples, and the circles and squares are daytime and nighttime samples, respectively. The arrowed points correspond to the spectra in Fig. 1.

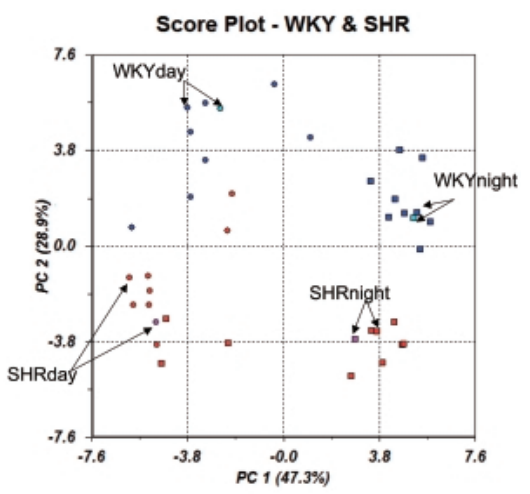

Fig. 3 PCA score plot of 39 data including 4 re-measured data points. The pairs of original and re-measured data are indicated by arrows

with $\pm 0.02 \mathrm{ppm}$ tolerance, respectively, from the $0.04 \mathrm{ppm}$ width of bucket integration. Figure 5 shows them on the spectra. The peak of $2.41 \mathrm{ppm}$ might be identified as succinate, 2.58 and $2.70 \mathrm{ppm}$ might be assigned to two peaks out of the citrate 4 peaks and the peak of $3.27 \mathrm{ppm}$ might be assigned to be TMAO (trimethylamineoxide). ${ }^{10}$

Likewise, regarding the classification of (2), all of the daytime and nighttime samples were made as independent mathematical models by PCA, and then SIMCA. In Fig. 6, the Cooman's plot shows that samples of daytime and nighttime were almost in their $68 \%$ confidence limit, respectively, which also means that good classification resulted. The values of the discrimination power in Fig. 6 are mostly higher than those in Fig. 4, which means that the spectral profiles of daytime and nighttime have relatively large differences, which is unexpected. In this case, we selected a variable set with discrimination powers higher than 3.0 in Fig. 7, which are 1.32, 1.36, 2.52, 2.68, 3.82 and $9.12 \mathrm{ppm}$, with $\pm 0.02 \mathrm{ppm}$ tolerances same as above case, respectively. This is a different variable set from that between WKY and SHR models. The peaks of 1.32 and $1.36 \mathrm{ppm}$ might be assigned to be lactate, the peaks of 2.52 and $2.68 \mathrm{ppm}$ might be assigned to be two peaks of the citrate, the peak of $3.82 \mathrm{ppm}$

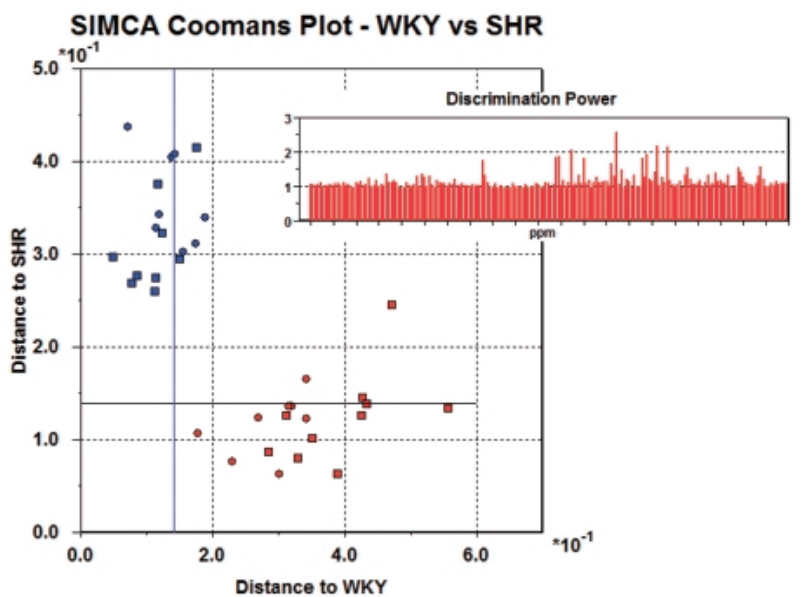

Fig. 4 Cooman's plot and discrimination power plot via 188 variables (chemical shifts) for WKY and SHR rat model. Blues and reds indicate WKY and SHR samples, respectively. Cooman's plot describes class boundaries, the $68 \%$ confidence limit of each model by horizontal and vertical bars.

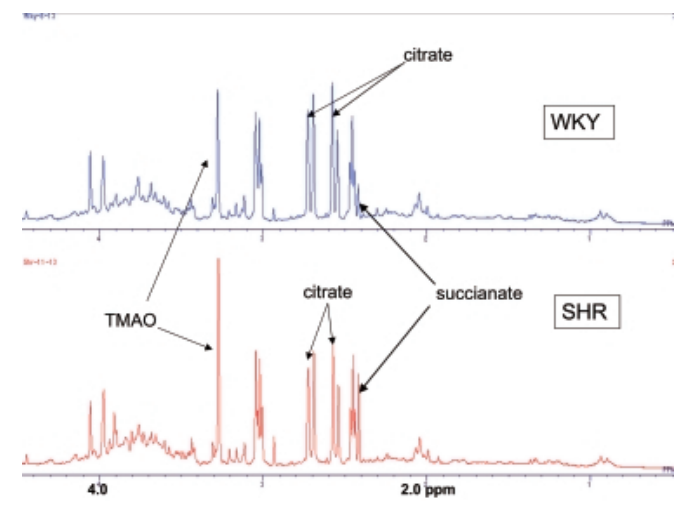

Fig. 5 Two example ${ }^{1} \mathrm{H}$ spectra from each of WHY and SHR. Several highest discrimination powers in Fig. 4 are indicated on the night spectra. The arrows indicate succianate, citrate, and TMAO (trimethylamineoxide) peaks, respectively.

could not be definitely identified with overlapped signals, and the small peak of $9.12 \mathrm{ppm}$ might tentatively be assigned to be $N$-methylnicotinic acid. ${ }^{11}$

\section{Discussion}

This report is an analysis of successful classification for reflecting the metabolic difference of urine between WKY and SHR and between daytime and nighttime. Considering the latter finding, reports concerning the metabolic response, during in the past several years, did not pay sufficient consideration on the time of day of the sampling. A biochemical examination, as well, should also perform during the same time range of a day.

In two comparisons (shown in Figs. 4 and 6), the different combinations of marker variables revealed here suggest that there are different mechanisms for maintaining homeostasis in living systems by means of excretions as buffers.

Regarding the difference between WKY and SHR, this might be caused by innate multi-factors of each strain, though there is not enough evidence to correlate only to hypertension itself. 


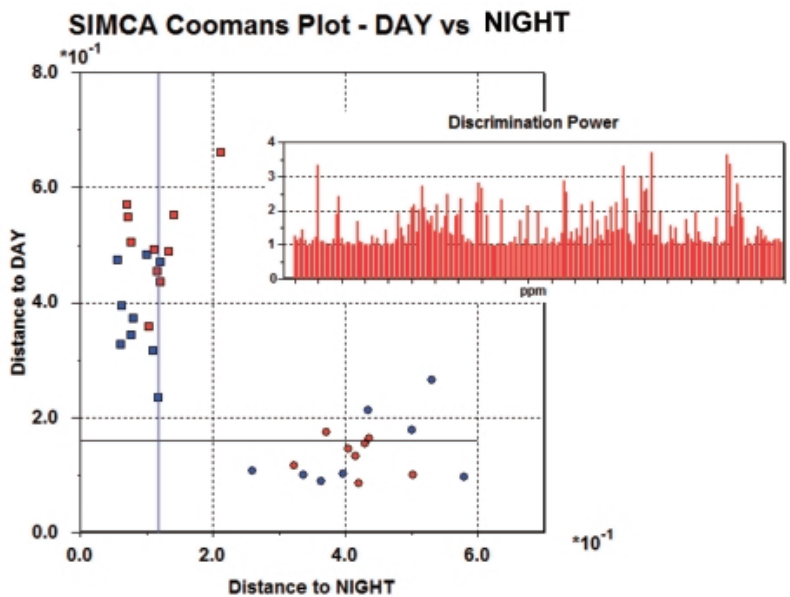

Fig. 6 Cooman's plot and discrimination power plot via 188 variables (chemical shifts) for daytime and nighttime rat models. The circles and squares indicate daytime and night samples, respectively. The horizontal and vertical bars in the Cooman's plot are the same as in Fig. 4.

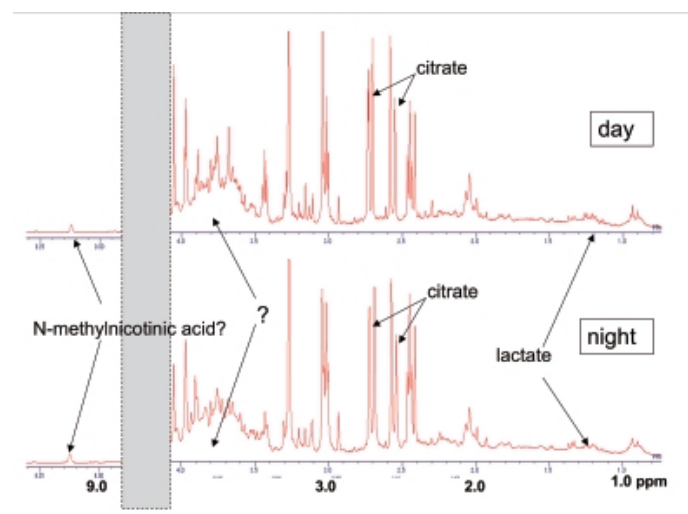

Fig. 7 Two example ${ }^{1} \mathrm{H}$ spectra from daytime and nighttime samples. Lactate, citrate and $N$-methylnicotinic acid are indicated by arrows on the spectra; the peak of $3.82 \mathrm{ppm}$ is not definitely identified. The gray box indicates a skip of horizontal scale.

For a reliable statistical analysis, more datasets should be accumulated. In our laboratory, further measurements of data at various times of the day are planned in order to reveal timerelated phenomena e.g. a circadian rhythm, various strains of both normal and disease model analysis to focus candidates for the marker compound directly with a correlated hypertensive disease.

As mentioned in the introduction, the assignment or identification of each peak for the ${ }^{1} \mathrm{H}$ spectra is not necessary in bio samples, and consequently the analysis concluded unsuspected marker variables correlated to the innate symptoms. In Figs. 4 and 6, the comprehensive NMR measurement captures the synchronous marker variables to have a dominant correlation in the total balance change of urine samples. It is noted that an individual variable change, itself, has less direct meaning independently. We selected the highest marker variables, as shown in Figs. 5 and 7 . The threshold value of the discrimination power has no definite criterion, however, and depends upon the characteristics of the samples; especially, the entire profile of the discrimination power plot should be taken into account for a further investigation. This is the essential point of difference from other separation analysis methods, such as mass spectroscopy.

In this detective way of markers by the SIMCA method, some overlapped and/or spin-coupled peaks were identified tentatively and ambiguously; consequently, ultimate 2D-NMR techniques, such as COSY, HMQC, or DOSY, will add the correct dimension to resolve the overlapped signals and give additional information analysis of the marker compound.

To summarize this report, our experiment on NMR measurements coupled with the pattern recognition method offered clear classification reflecting the inherent metabolic information of strains or diurnal variation, and also a preliminary screening test for marker candidate variables out of multi-component urine samples. NMR-based metabolomics has many advantages: easy preparation for samples, comprehensive capturing of proton-containing compounds, as well as modern developmental NMR techniques ready to be facilitated. Thus, this analysis here would provide a fundamental and powerful tool for perspective investigations of disease metabolism.

\section{References}

1. J. K. Nicholson, J. C. Lindon, and E. Holmes, Xenobiotica. 1999, 29, 1181.

2. J. C. Lindon, J. K. Nicholson, E. Holmes, H. Antti, M. E. Bollard, H. Keun, O. Becknert, T. M. Ebbels, M. D. Reily, D. Robertson, G. J. Stevens, P. Luke, A. P. Breau, G. H. Canter, R. H. Bible, U. Niederhauser, H. Senn, G. Schlotterbeck, U. G. Sidelmann, S. M. Laursen, A. Tymiak, B. D. Car, L. Lehman-Mcleeman, J. M. Colet, A. Loukaci, and C. Thomas, Toxcol. Appl. Pharmacol., 2003, 186,137.

3. J. D. Bell, P. J. Sadler, V. C. Morris, and O. A. Levander, Mag. Res. in Med. 1991, 17, 414.

4. M. E. Bollard, E. G. Stanley, J. C. Lindon, J. K. Nicholson and E. Holmes, NMR Biomed., 2005, 18, 143.

5. E. Holmes, A. W. Nicholls, C. Lindon, S. Ramos, M. Spraul, P. Neidig, S. C. Connor, J. Connely, S. J. P. Dammets, J. Haselden, and J. K. Nicholson, NMR Biomed., 1998, 11, 235.

6. Released on Oct. $\mathbf{2 0 0 5}$ from JEOL Ltd.

7. B. Kowalski, D. Sharaf, and D. Iliman, "Chemomtrics", 1986, John Wiley and Sons, New York.

8. S. Wold, Pattern Recognition, 1976, 8, 127.

9. S. Wold and M. Sjostrom, "SIMCA: Method for Analyzing Chemical Data in Terms of Similarity and Analogy", in "Chemometrics: Theory and Application", ed. B. R. Kowalsky, 1977, American Chemical Society, Washington D.C., 243.

10. J. K. Nicholson and I. D. Wilson, Prog. NMR Spectrosc., 1989, $21,449$.

11. T. W. -M Fan, Prog. NMR Spectrosc., 1996, 28, 161. 